\title{
Increasing the autonomy of Wireless Sensor Node by effective use of both DPM and DVFS methods
}

\author{
Van-Trinh HOANG, Nathalie JULIEN, Pascal BERRUET \\ van-trinh.hoang@univ-ubs.fr, nathalie.julien@univ-ubs.fr, pascal.berruet@univ-ubs.fr \\ Lab-STICC Research Center, University of South-Brittany, BP 92116, 56321 Lorient, France.
}

\begin{abstract}
The use of ubiquitous computing devices like Wireless Sensor Networks (WSNs) has increased with an exponential growth in recent years, due its low-cost, portability, easy deployment, self-organization. Since WSNs are battery-powered devices, thus main challenge faced by designers is reduction of energy consumption in WSNs. This paper presents an effective implementation of Dynamic Power Manager (DPM) and Dynamic Voltage and Frequency Scaling (DVFS) technics to increase the autonomy of wireless sensor node by reducing dynamic power dissipation. Simulation with the application of hazardous gas detection using Capnet-PE tool indicates significant energysaving, which allows us to evaluate and validate our approach for WSN.
\end{abstract}

Index Terms: energy-efficiency, Power Availability Manager (PAM), FPGA, Dynamic Power Manager (DPM), Dynamic Voltage and Frequency Scaling (DVFS).

\section{INTRODUCTION}

The wireless sensor networks consist of small and inexpensive sensor nodes with some computational power, sensing and storage capability. They are intended for a broad range of application domains to cooperatively monitor physical or environment conditions such as temperature, sound, pressure, hazardous gas, air pollution, intrusion of person at different locations. In several applications, a large number of sensor nodes is deployed in order to achieve high quality, faulttolerance sensing network. However, the lifetime of sensor nodes are attached to a limited battery, thereby it is desirable to make these nodes as energy-efficient as possible to maintain high quality results. In other words, we try to reduce the energy consumption of sensor nodes to extend their lifetime. There are two primary types of power dissipations in electronic devices: dynamic and static.

The dynamic power is associated with the switching of logic states caused by the active computations, which is central to perform logic operations. While static currents occur whenever the elements are switched on to hold or maintain the logic states between switching events, even when any computation is not carry on them. Therefore, power static is wasted because it does not contribute to computation; unfortunately this power leakage is unavoidable. Two major solutions of energy-saving are DPM (Dynamic Power Manager) and DVFS (Dynamic Power and Frequency Scaling). DPM aims to reduce the static power by turning off a part circuit or running it in low-power modes when any workload is not experienced. DVFS deals with the dynamic consumption by adjusting supply voltage and operating frequency to meet application requirement. However, only few publications address the use of both DPM and DVFS in Wireless Sensor Node(WSNO).

This paper contributes an effective use of both DPM and DVFS methods to maximize energy-saving in WSNO. The remainder of this paper is as follows. Section II describes two major technics: DPM and DVFS for reducing power dissipation. Then section III presents the hardware model of our sensor node. Next, section IV depicts the implementation of efficient use of both DPM and DVFS in wireless sensor node. The last two sections give our evaluation and conclusion.

\section{Methods OF ENERGY REDUCTION}

The total power dissipation in sensor node is the sum of static power and dynamic power as follows:

$P_{\text {tot }}=P_{\text {static }}+P_{\text {dynamic }}=\left(P_{\text {leakage }}+P_{\text {bias }}\right)+\left(P_{s c}+P_{\text {sw }}\right)$

The static power $P_{\text {static }}$ has two parts: leakage power $P_{\text {leakage }}$ and bias power $P_{\text {bias }}$. While the dynamic power $P_{\text {dynamic }}$ consists of short-circuit power $P_{s c}$ and switching power $P_{s w}$. Among these four sources of power dissipation, leakage power $P_{\text {leakage }}$ and switching power $P_{s w}$ are currently the dominant ones which account approximately more than $95 \%$ the total power consumption. Several solutions of hardware design are introduced in [2] to reduce $P_{\text {leakage. }}$ In our work, the energy consumption $P_{s w}$ is decreased using both DPM and DVFS technics, the first one reduces the static power by turning off a part circuit or running it in low-power modes when there is no workload, whereas the second lower the dynamic power. The computation of dynamic power for performing the task $t_{a}$ is given as follow:

$$
P_{\text {dynamic }}^{\text {ta }}=\alpha \cdot C_{\text {load }} \cdot V_{d d}^{2} . f
$$

Where $\alpha$ is switching activity, thus its energy consumption during a time interval $t=N_{C}^{t a} \cdot T$, is computed as following equation:

$$
E_{\text {dynamic }}^{t a}=N_{C}^{t a} \cdot \alpha \cdot C_{l o a d} \cdot V_{d d}^{2}
$$

Considering the Eq.(2), decreasing operating frequency leads to a reduction in power dissipation, but it does not reduce the energy consumption (Eq.(3)). Hence, the only posibility to reduce the energy consumption is to reduce the circuit supply voltage $V_{d d}$. Of course, reducing the supply voltage necessitates the reduction of operating frequency in order to 
ensure correct operation. Several strategies can be applied for the use of DVFS method:

- All the tasks are supplied with the same low voltage to reduce the energy dissipation.

- Each task is supplied with a different voltage based on the rate of its activity.

- The first task is run with the highest frequency, i.e. with the highest voltage. Based on the actual execution time of this task, the voltage and frequency are regulated consistently for the next task, and so on as described in [3].

The next section presents hardware description of our sensor node including additionally intelligent element named Power Availability Manager (PAM), so as to manage the effective use of DPM and DVFS solutions.

\section{WIRELESS SENSOR NODE MODEL}

The wireless sensor node interfaces with the environment using actuators and sensors, and communicates with other nodes via wireless links. The original configuration of wireless sensor node includes processor, memory, Interface for Actuator and Sensors (IAS), Radio Transceiver Module (RTM), battery and DC-DC converters(power switches) (as shown in Fig.1). In many applications, wireless sensor networks are deployed in harsh environment such as forest fire detection, earthquake warning, hazardous gas detection, enemy movement tracking in secured zone, etc, therefore it is difficult for human intervention in case of energy depletion. For this reason, our node is supported by a novel device including Power and Availability Manager (PAM) block combined with FPGA, their functionality is described as follows:

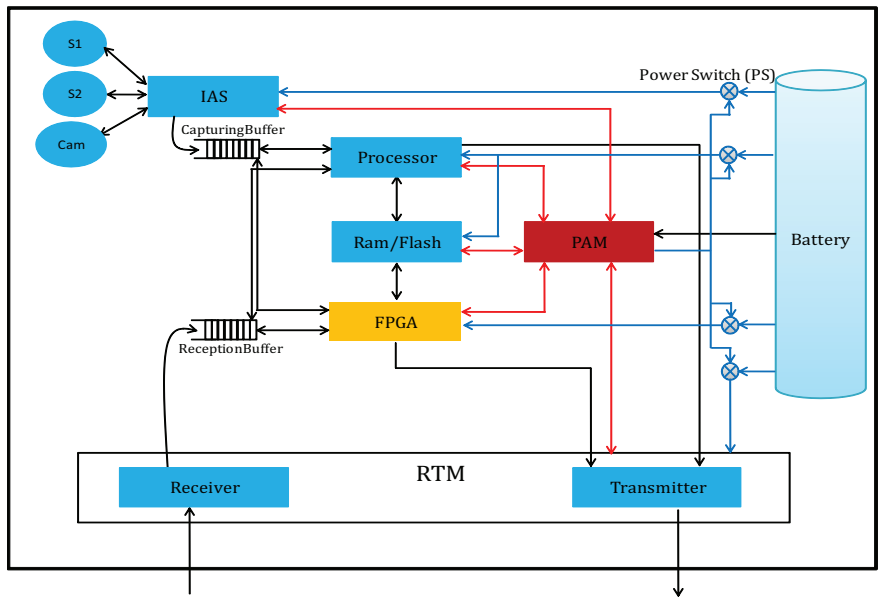

Fig. 1. Hardware configuration of wireless sensor node

- Power Availability Manager (PAM) is intelligent part of our sensor node that manages efficiently the energy consumption in sensor node. Furthermore, our PAM block also supports the node to detect the failing element, and then take an appropriate corrective solution, which is described in previous works [4-5].

- Configurable zone of FPGA is included in sensor node to increase the availability of sensor node and enhance the node performance for signal and video processing. Our PAM block can be resided into FPGA.

To mitigate the data conflict, two First In First Out (FIFO) buffers are used in which CapturingBuffer stores the capturing data, and ReceptionBuffer saves the data sent by other nodes.

\section{IMPLEMENTATION OF DPM AND DVFS IN WIRELESS SENSOR NODE}

In this section, we describe how to implement both DPM and DVFS methods to reduce energy consumption in wireless sensor node. As mentioned previously, DPM solution is used for minimize the static power when any workload is run by switching to very low-power modes (Sleep or Deep-Sleep), whereas DVFS method aims to lower energy dissipation when application tasks are executed by ajusting dynamically supply voltage and operating frequency. As explained in [1], the use of DVFS method comes at expense of power overhead due to its complex computation. To address this issue, only two values of supply voltage and corresponding operating frequency are selected according to the type of received events (as described in Fig.2), in which:

- Node elements (processor, memory, radio module) are supplied with low voltage and correspondingly low frequency when received data containing no hazardous event.

- Otherwise, when a hazardous event arrives, these elements are powered with high voltage and operates at correspondingly high frequency.

The adjustment of supply voltage and operating frequency in sensor node for each node element such as processor, memory and radio transceiver is undertaken by PAM block. The next subsections describe the implementation of DVFS method into each node element.

\section{A. Implementation of DVFS in processor and memory}

Initially, the processor and memory of sensor node are powered with low voltage and operate at correspondingly low frequency to realize normal sensings. However, when sensing data contains hazardous event, the PAM block regulates the supply voltage of these elements to maximum. That accelerates the speed of data processing to prepare data packet for radio transmission.

\section{B. Implementation of DVFS in radio module}

In our wireless sensor network, multi-hops communication is used to send gathering data to the base station, which allows to balance the energy consumption among all the nodes in the network. For example, wireless communication between the sensor nodes is illustrated in Fig.3.a, node $A->\operatorname{node} B->$ node $C->$ BaseStation when node A transmits a data containing no hazardous event to the base station through nodes B and C. In this case, radio transceiver is powered with low voltage and low frequency, i.e. it operates lower data throughput. However, if a hazardous event is detected in node A, the supervisors have to be informed as soon as possible such that a decision is promptly given. Hence, the data might be sent quickly to base station with less hops number as depicted 


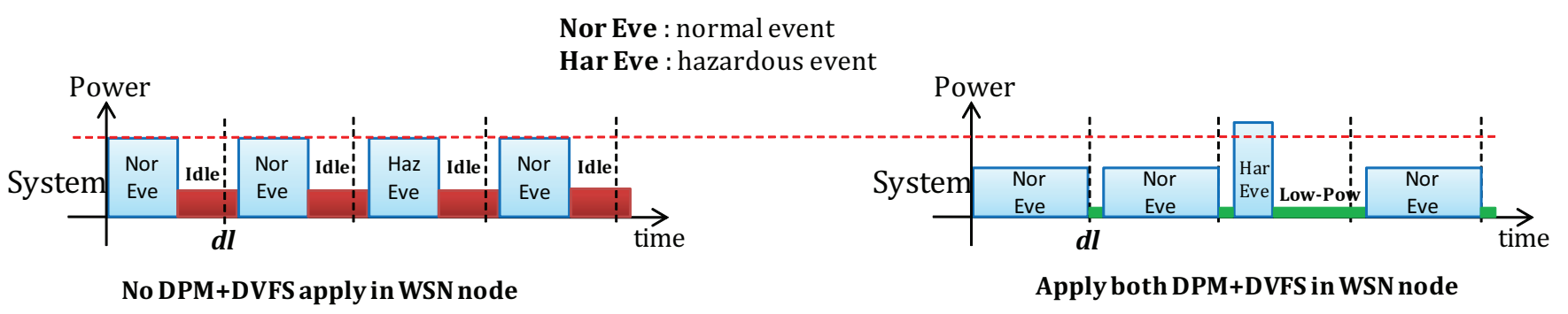

Fig. 2. Implementation of DPM and DVFS methods in wireless sensor node

in Fig.3.b, which increases the dissipated energy that allows to communicate with further sensor node. In this case, radio transceiver is supplied with high voltage and attached high frequency that also increases the data throughput. The relation between transmitter energy and communication distance is illustrated in Eq.4.

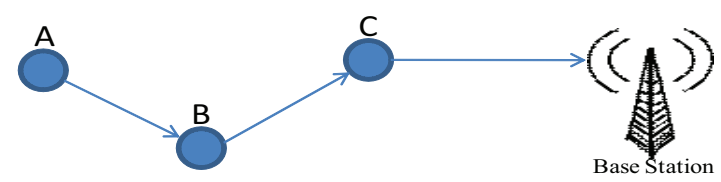

a.

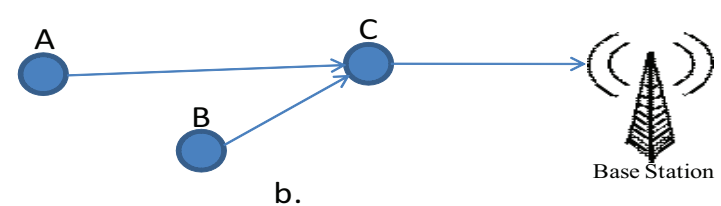

Fig. 3. Data transmission between WSNO

$$
E_{T x}(k, d)=E_{E l e c} \cdot k+\alpha_{a m p} \cdot k \cdot d^{2}
$$

Where the radio dissipates $E_{\text {Elec }}$ per bit to run transmitter circuitry, and $\alpha_{a m p}$ is the transmission amplifier. Hence to transmit a $k$-bit data packet a distance $d$ using radio communication, the necessary energy consummed in radio is $E_{T x}(k, d)$. The next section provides our evaluation of implementation DPM and DVFS solutions into wireless sensor node.

\section{Evaluation}

To evaluate energy-efficiency when applying DPM and DVFS methods in wireless sensor node, an application of hazardous gaz detection for area such as harbor or warehouse, which is developed by our partner, ERYMA Company [8], is used. The energy dissipation of sensor node is then simulated using CAPNET-PE simulator [6]. This tool allows us to estimate the consumption of sensor node based on a scenario of event generation. The original sensor node consists of main elements as listed in the Table I, the power switches (LM3100 and MAX618), and a battery. The consumptions of elements such as PAM block combined with FPGA are also simulated. In our study, the FPGA IGLOOV2 of Actel manufacturer will be selected to include PAM due to its reliability and ultralow power consumption [7]. The power consumption of each element with corresponding supply voltage is given in the Table I.

TABLE I

POWER CONSUMPTION OF EACH ELEMENT IN WSNO

\begin{tabular}{|c|c|c|}
\hline Element & Supply voltage (V) & Power (mW) \\
\hline PIC24FJ256GB110 & 3.3 & 51 \\
\hline M48T35AV memory & 3.3 & 150 \\
\hline Oldham OLCT 80 & 20 & 867 \\
\hline Miwi Radio transmitter & 3.3 & 130 \\
\hline Miwi Radio receiver & 3.3 & 70 \\
\hline
\end{tabular}

To apply DVFS method without suffering large energy overhead due to its complex computation, two supply voltages of $3 \mathrm{~V}$ and $3.6 \mathrm{~V}$ are selected for processor, memory and radio module. These two voltage values correspond to the minimum and maximum functioning voltage noted in the datasheets of these elements. Based on the Eq.2, their new values of power dissipation are computed as follow:

$$
P_{t}=\frac{V_{t}^{2}}{V_{3.3}^{2}} \cdot \frac{f_{t}}{f_{3.3}} \cdot P_{3.3}=\frac{V_{t}^{2}}{V_{3.3}^{2}} \cdot \frac{1}{e} \cdot P_{3.3}
$$

Where $P_{t}$ and $V_{t}$ are respectively the actual power consumption and supply voltage, and $e=\frac{T_{t}}{T_{33}}$ is extension factor between the new and nominal time execution $\left(e_{3 / 3.3}=1.17\right.$ and $e_{3.6 / 3.3}=0.87$ in our study). Tow cases with duration of one day are realized by using CAPNET-PE tool in function of the rate of generation of event in the network. Three cases are simulated as follow:

- Neither PAM block combined with FPGA nor two methods of DPM and DVFS are used.

- Only use of PAM block combined with FPGA, and DPM method.

- Both use of PAM block combined with FPGA, DPM and DVFS methods.

The energy consumptions of processor, memory and radio module are respectively depicted in Fig.4, Fig.5, and Fig.6. These figures indicate that when DPM method is used, a large significant energy-saving up to $75 \%$ for processor, and $32 \%$ for memory when the number of event is high (240 events per day), because the consummed powers when these elements are in Idle mode $\left(P_{\text {IdlePro }}=38.4 \mathrm{~mW}\right.$ and $P_{\text {IdleMem }}=13.2 \mathrm{~mW}$ ) are much higher than the powers when they are in Deep-Sleep mode $\left(P_{\text {DeepSleepPro }}=5 \mathrm{~mW}\right.$ and $P_{\text {DeepSleepMem }}=1 \mathrm{~mW}$ ). The simulation results of third case still show an improvement in energy reduction compared to the 
second case, when energy gains are up to $80 \%$ for processor, $53 \%$ for memory, and $31 \%$ for radio module.

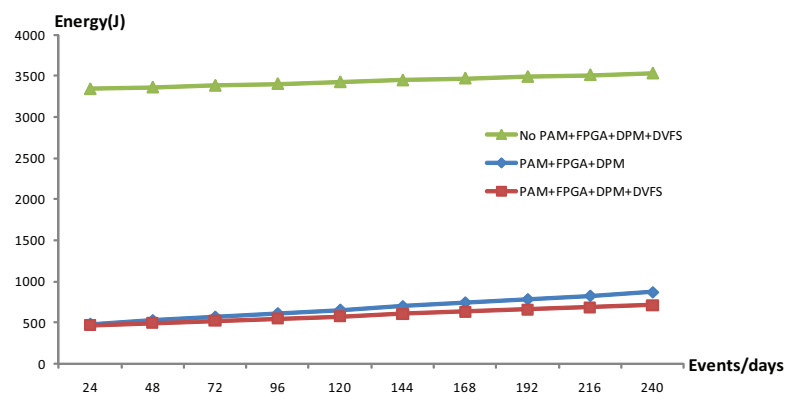

Fig. 4. Energy consumption of processor

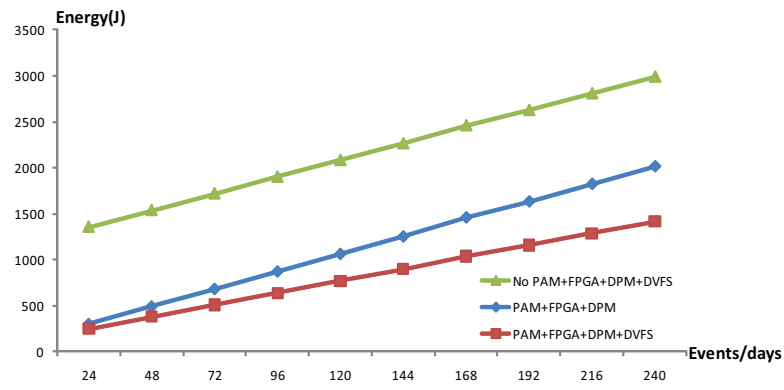

Fig. 5. Energy consumption of memory

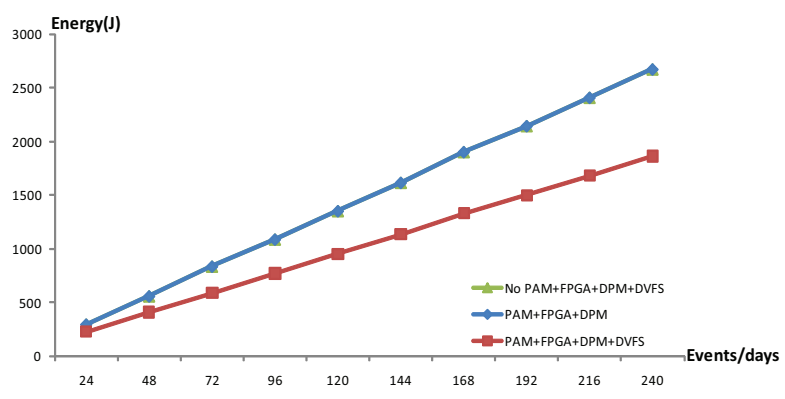

Fig. 6. Energy consumption of radio transceiver

The Fig.7 shows the percentage of energy reduction using DPM method or both DPM and DVFS methods in wireless sensor node, in which energy gains are up to $69 \%$ and $72 \%$ when number of event is low ( 24 events per days). But these gains are degraded when the number of event is high, only $25 \%$ and $35 \%$ of energy reduction, because the gas sensor of node, which is the most critical element in term of energy consumption (as listed in Table I), is enabled more times to sense data than the cases of lower number of event. This energy dissipation is unavoidable. However, we also show the interest of implementing DPM and DVFS methods in sensor node through the energy gains in node elements such as processor, memory and radio module. Additionnaly, the consumption of both PAM block and FPGA is very small as depicted in Fig.8, which makes them suitably to implement into sensor node.

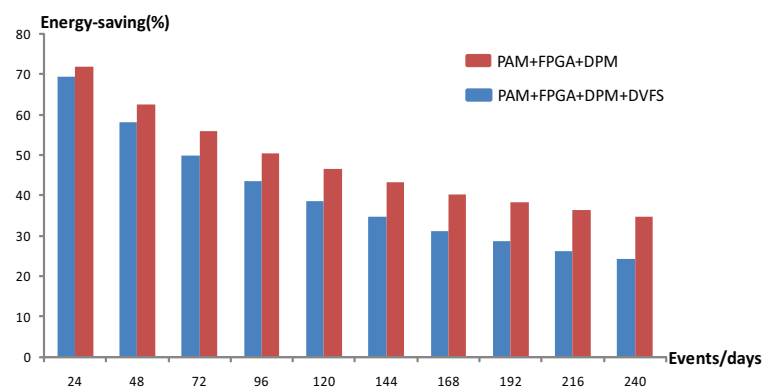

Fig. 7. Percentage of energy saving using DPM and DVFS in WSNO

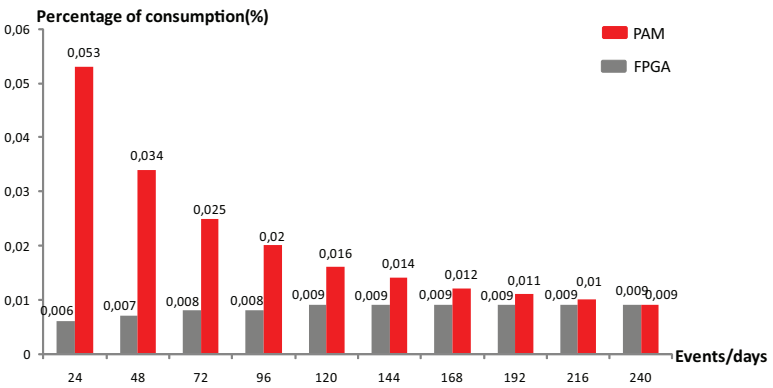

Fig. 8. Consumption percentage of PAM and FPGA in WSNO

\section{CONCLUSION}

This paper introduces an effective way of using both DPM and DVFS methods in WSNO to reduce energy dissipation, which leads to increase the battery life, i.e. the autonomy of wireless sensor node. The simulation results with Capnet-PE tool show that our approach significantly increases the energysaving of node that allows to validate our work. Our future work will verify the impact of using DPM and DVFS in sensor node on WSN. And our approach will be implement on a real case in order to validate with the measurements.

\section{REFERENCES}

[1] Marcus T. Schmitz, Bashir M. Al-Hashimi, Petru Eles, System-Level Design Techniques for Energy-Efficient Embedded Systems, Kluwer Academic Publishers, first edition, Boston, USA, 2004.

[2] B.S. Deepaksubramanyan and Adrian Nunez, Analysis of Subthreshold Leakage Reduction in CMOS Digital Circuits, PROCEEDINGS OF THE $13 \mathrm{TH}$ NASA VLSI SYMPOSIUM, POST FALLS, IDAHO, USA, JUNE 5-6, 2007.

[3] Bhatti, M.K., Belleudy, C. and Auguin, M., An inter-task real time DVFS scheme for multiprocessor embedded systems, IEEE Conference on Design and Architectures for Signal and Image Processing (DASIP), Edinburgh, England, October 26-28, 2010.

[4] V.T. Hoang, N. Julien and P. Berruet, Design under Constraints of Availability and Energy for Sensor Node in Wireless Sensor Network, IEEE Conference on Design and Archtectures for Signal and Image Processing, Karlsruhe, Germany, October 2012.

[5] V.T. Hoang, N. Julien and P. Berruet, On-line self-diagnosis based on power measurement for a wireless sensor node, First Workshop on Highly-Reliable Power-Efficient Embedded Designs, Shenzhen, China, February 2013

[6] Nicolas Ferry, Sylvain Ducloyer, Nathalie Julien and Dominique Jutel, Power/Energy Estimator for DesigningWSN Nodes with Ambient Energy Harvesting Feature, EURASIP Journal on Embedded Systems, January, 2011.

[7] http://www.actel.com/documents/IGLOO.

[8] http://www.eryma.com. 